\title{
Multiple Clustered and Focally Atrophic Dermatofibromas: Case Report and Literature Review
}

\author{
Gustavo Moreira Amorim ${ }^{1,2}$, Eduardo Dan Itaya ${ }^{1}$, Gabriela Coelho Itaya ${ }^{1}$, Luiza Mousquer Leal ${ }^{1}$, Amanda \\ Amaro Pereira ${ }^{3}$, Roberto Moreira Amorim Filho ${ }^{2}$
}

${ }^{1}$ Faculty of Medicine, South Santa Catarina University

${ }^{2}$ Sector of Dermatology, Santa Teresa Hospital, Santa Catarina State Secretary for Health

${ }^{3}$ Polydoro Ernani de Sao Thiago University Hospital - Santa Catarina Federal University

Received: May 08, 2020 ; Accepted: May 14, 2020; Published: May 25, 2020

*Corresponding author: Gustavo Moreira Amorim, MD, Msc, 135 Duarte Schutel street, downtown, Florianópolis, Santa Catarina, Brazil, Tel. No: +5548999827097; E-mail: gustavomoreiraamorim@hotmail.com

\begin{abstract}
We present a case of a 50 year-old woman with multiple erythematous and hyperpigmented plaques, with achromic and slightly atrophic centers, restricted to a body segment. Dermoscopy showed a pigmented network surrounding a central scar-like patch. Histopathology, complemented by immunohistochemical profile was compatible with dermatofibromas, arranged in a multiple clustered form. Relevant literature on the topic was reviewed.
\end{abstract}

Meshterms: Factor 13a Positivity; Benign Fibrous Histiocytoma;

Multiple clustered dermatofibroma; Segmental Distribution

\section{Introduction}

Dermatofibromas, also known as fibrous histiocytomas, are a benign proliferation of oval cells resembling histiocytes along with spindle-shaped cells, resembling fibroblasts and myofibroblasts [1]. Etiology of dermatofibromas remains uncertain. Literature, supported by cytogenetic studies identifying clonality, considers it to be a benign neoplastic lesion. Classically, it was attributed to the occurrence of previous trauma to the skin, such as arthropod bites, however this has never been proven [1-3]. Usually, dermatofibromas are painless dermal nodules, yellow-brown in colour and slightly scaly,commonly present as small, single lesions on the lower limbs of middle-aged women, although bigger lesions are occasionally seen, mainly in the cellular and aneurysmal histological subtypes. Multiple lesions may develop [1]. We present an emblematic case of a 50 year-old woman with multiple erythematous and hyperpigmented plaques, with achromic and slightly atrophic centers, restricted to a body segment, which the histopathologic investigation confirmed to be dermatofibromas.

\section{Case report}

A 50-year-old woman, with hypertension treated with losartan (50mg, twice a day) was admitted to the outpatients' clinic due to abdominal lesions. She reported the appearance of nodules and plaques on the abdomenin her adolescence; at that time they quickly progressed in number and later stabilized, with local pruritus. She denied inflammation or previous trauma. On physical examination, there were asymmetrically distributed lesions on the right lower quadrant of the abdomen, consisting ofbrownish hyperchromic papules and plaques, some with overlying erythema, and rough texture. The adjacent skin clearly showed atrophy. Palpation also suggested atrophy, with the feeling of herniated skin similar to anetoderma (Figures 1 and 2).
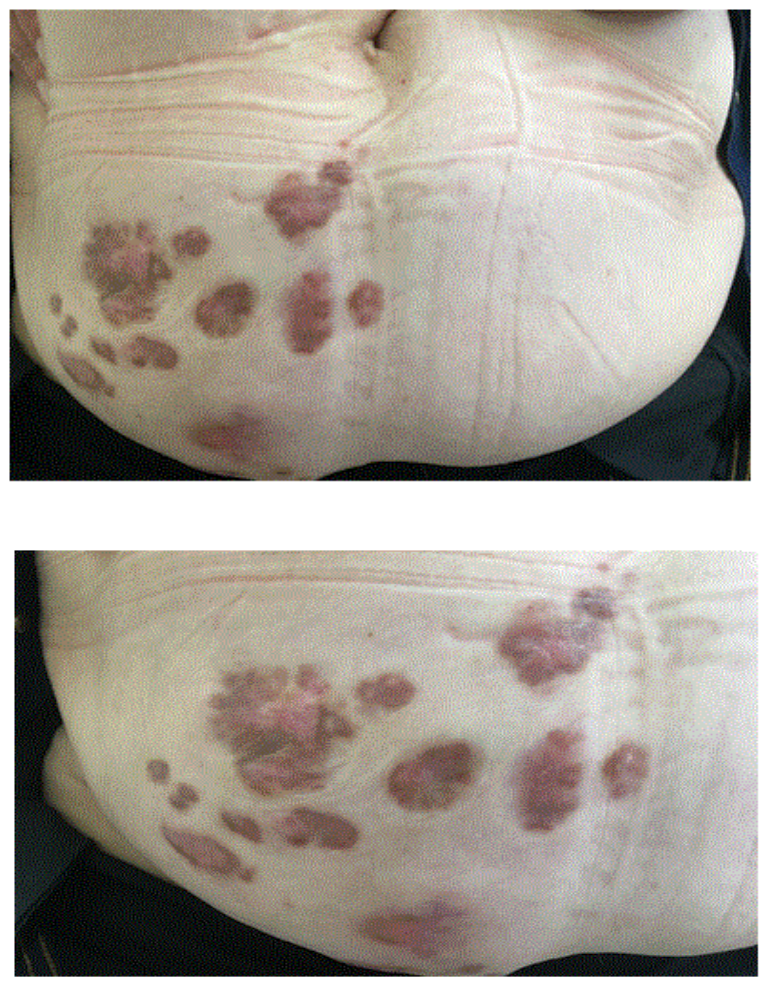

Figures 1 and 2: Photography of brownish hyperchromic papules and plaques asymmetrically distributed on the right lower quadrant of the abdomen 
A fusiform excision of the skin was performed with the following hypotheses: sarcoidosis, necrobiosislipoidica and extragenital lichen sclerosusetatrophicus.The histopathological examination showed, in the dermis, aproliferation of spindled, dendritic cells, permeating collagen bundles, which were hyalinized and thickened, amid capillaries and histiocytes. The overlying epidermis exhibited mild acanthosis, with areas of basal hyperpigmentation. Due to the histopathological hypothesis of dermatofibroma, immunohistochemical examination was performed, with positivity of Factor XIIIa in the dendritic cells, which were negative for CD34. HHV8, desmin and S100 were also negative. This immunohistochemical profile was compatible with dermatofibroma (Figures 3-7).

After the result of the histopathological examination, dermoscopic examination revealed a white amorphous area surrounded by a structure mimicking pigment network.Taking into account the clinical, histopathological and immunohistochemical aspects, this condition was established as multiple clustered dermatofibromas, with focal atrophy associated.

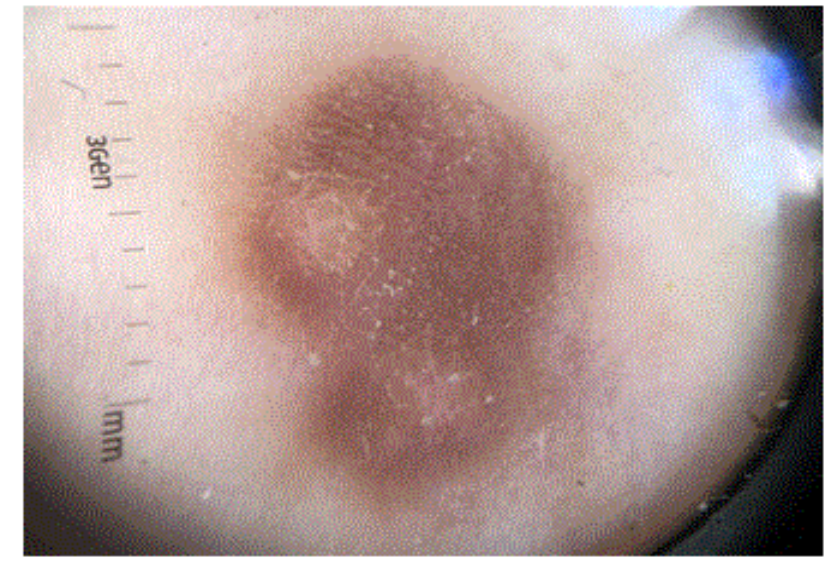

Figure 3: Dermoscopy reveals a white amorphous area, surrounded by a structure mimicking pigment network.

\section{Discussion}

Multiple clustered dermatofibromas a term used to describe the presence of multiple dermatofibromas clustered in a single anatomic region. It is a rare condition, first described by Dupre et al. in 1984 [4], with about 20 cases reported to date. Most of the reported cases were in females in the second and third decades of life [4-6]. Clinically, these lesions appear as multiple nodules grouped and arranged in a linear fashion located in only one anatomical site, usually asymptomatic and in the lower half of the body [5-7]. In this report, the patient was female and her condition started during adolescence, with lesions presenting as multiple brownish hyperchromic papules and plaques in only one anatomical site, like the ones described in the literature. However, her lesions presented with pruritus and were located in the abdominal region, unlike the previously reported cases.
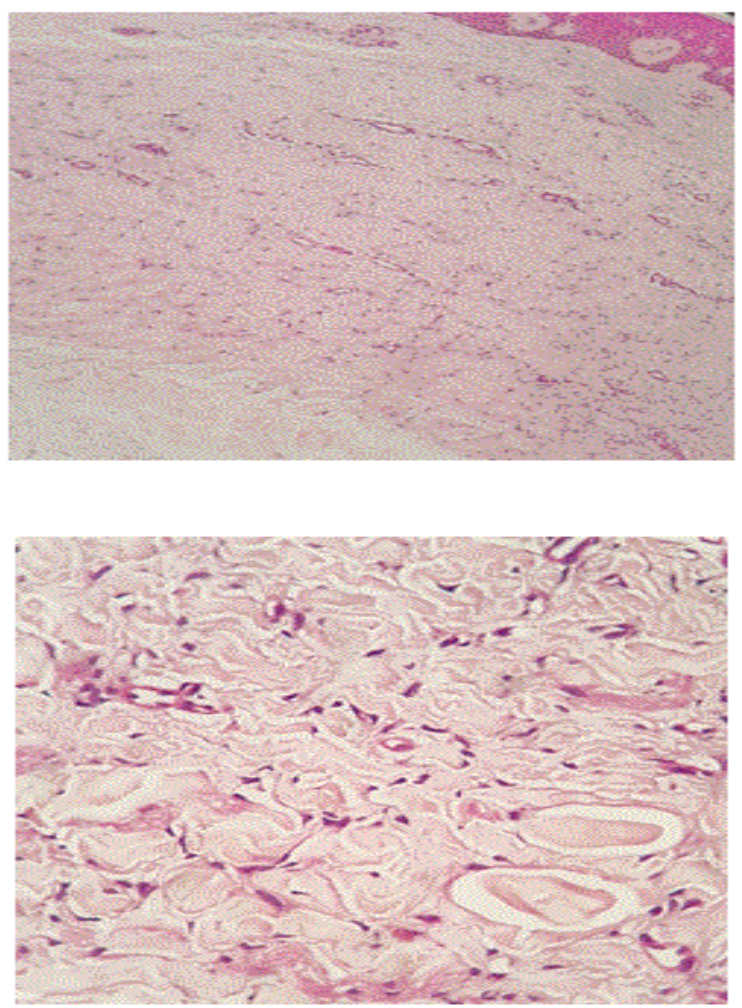

Figures 4 and 5: Hematoxylin-eosin, 100x and 400x: proliferation of spindle cells permeating thickened collagen bundles, amid capillaries and histiocytes
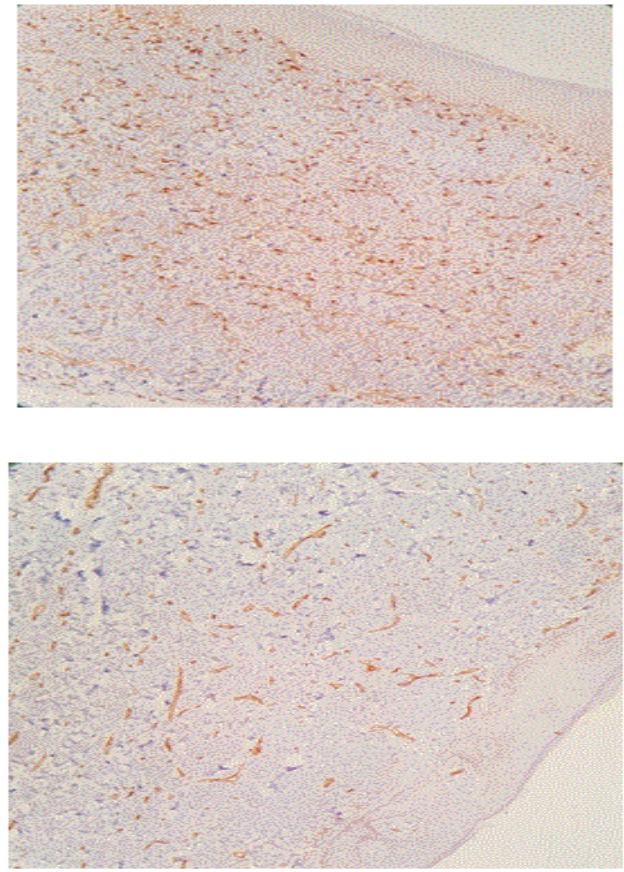

Figures 6 and 7: Positivity of the cells for Factor XIIIa (6); Negativity for CD34 (7), which stained the vessel walls only. 
The palpation of the lesions suggested atrophy, which is clearly observed in the adjacent skin. The atrophic variant represents $2 \%$ of all dermatofibromas $[8,9]$. There are multiple dermoscopy patterns that can be found in Dermatofibromas. Dermoscopy of the present case showed a white amorphous area, surrounded by apseudo-pigmented network structure, being the classic presentation of solitary dermatofibromas [10-12]. The diagnosis begins with the clinical and physical examination of the patient and is followed by a biopsy for histopathological evaluation. In this case, the histopathological examination showed the classic findings of dermatofibroma, which were supported by staining of the cells for Factor XIIIa, and negativity of the other markers [1]. As for the treatment, the most indicated is outpatient monitoring. That is because although techniques such as cryotherapy, intra-lesional steroids, surgery and phototherapy have been researched, their success is still variable. In the case of surgery, the lesions end up recurring, so they are only recommended in cases of discomfort and/or aesthetic complaints reported by the patient $[5,9,13,14]$. In agreement with the patient, we opted for observation and regularly follow-up.

\section{Conclusion}

Multiple clustered dermatofibromas is a rarely encountered clinical condition, reason why we report this illustrative and well documented case report. Until the present moment there are no reports of malignant degeneration and outpatient monitoringseems to be the most appropriated conduct.

\section{References}

1. Burns T, Breathnatch S, Cox N, Griffiths C. Rook's Textbook of Dermatology - 9a Ed. Oxford, UK. Wiley-Blackwell. 2016.

2. Chen TC, Kuo T, Chan HL. Dermatofibroma is a clonal proliferative disease. J. Cutan. Pathol. 2000;27(1):36-39.

3. Luzar B, Calonje E. Cutaneous fibrohistiocytictumours - an update. Histopathology. 2010;56(1):148-165.

4. Dupre A, Christol B, Martinez D. Histiocytofibromes multiples groupes (in French). Annales de dermatologie et de venereologie 1984;111:163-164.

5. Pinto-almeida T, Caetano M, Alves R, Selores M. Congenital multiple clustered dermatofibroma and multiple eruptive dermatofibromas unusual presentations. An Bras Dermatol. 2013;88(6 Suppl 1):63-66.

6. Higaki-Mori H, Yoshida Y, Hisaoka M, Nishigori C, Shindo M, Yamoto O. Unusual Congenital Multiple Clustered Dermatofibroma: First Reportes Case on the Face. Acta Derm Venerol. 2019;99(3):341-342.

7. Habif TP. Dermatologia Clínica: guia colorido para diagnóstico e tratamento - 5a edicao. 2012.

8. Hendi A, Jukic DM, Kress DW, Brodlan DG. Atrophic dermatofibroma: a case report and review of the literature. Dermatol Surg. 2002;28(11):1085-1087.

9. Reynolds H, Perry A, Satter EK. Multiple clustered and focally atrophic dermatofibromas (DF). Dermatol Online J. 2014;20(5):22612.

10. Soon SL, Howard AK, Washington CV. Multiple, clustered Dermatofibroma: a rare clinical variant of dermatofibroma. J Cutan
Med Surg. 2003;7(6):455-457.

11. Arias EM, Moscarella E, Buçard A, Moreno C, Lallas A, Longo C, et al. The Dermoscopic variability of dermatofibromas. J Am AcadDermatol. 2015;72(1 Suppl):S22-24.

12. Shaheen B, Saldanha G, Calonje E, Johnston A. Multiple Clustered Dermatofibromas (fibrous histiocytomas): an atypical clinical variant of dermatofibroma. ClinExpDermatol. 2014;39(1):88-90.

13. Unamuno P, Carames Y, Lopez E, Hernandez-Martin A, Pena C. Congenital multiple clustered dermatofibroma. $\mathrm{Br} J$ Dermatol. 2000;142(5):1040-1043.

14. Villaverde R, Martinez MA, Cano SD. Multiple Clusteres Dermatofibromas Following Ustekinumab Treatment for Psoriases Vulgaris. Sultan Qaboos Univ Med J. 2017;17(3):e375-377. 